\title{
Prognostic value of surgical intervention in advanced lung adenocarcinoma: a population-based study
}

\author{
Shuncang Zhu^, Tao Ge, Junjie Hu, Gening Jiang, Peng Zhang \\ Department of Thoracic Surgery, Shanghai Pulmonary Hospital, Tongji University School of Medicine, Shanghai, China \\ Contributions: (I) Conception and design: S Zhu; (II) Administrative support: P Zhang; (III) Provision of study materials or patients: G Jiang, P \\ Zhang; (IV) Collection and assembly of data: S Zhu, T Ge; (V) Data analysis and interpretation: S Zhu, T Ge, J Hu; (VI) Manuscript writing: All \\ authors; (VII) Final approval of manuscript: All authors. \\ Correspondence to: Peng Zhang. Department of Thoracic Surgery, Shanghai Pulmonary Hospital, Tongji University School of Medicine, 507 \\ Zhengmin Road, Shanghai 200433, China. Email: zhangpeng1121@tongji.edu.cn.
}

\begin{abstract}
Background: Surgical intervention is generally not considered as a treatment option in patients with advanced non-small cell lung cancer (NSCLC). Accumulating data suggest that surgery may have beneficial effects for these advanced patients. However, no evidence supports the significance of primary tumor resection (PTR) and metastatic tumor resection (MTR) in patients with stage IV lung adenocarcinoma (LUAD).

Methods: A total of 32,497 patients diagnosed with primary stage IV LUAD were selected through the Surveillance, Epidemiology, and End Results (SEER) database. Possible confounders were eliminated by propensity score matching (PSM). The overall survival (OS) and lung cancer-specific survival (LCSS) were estimated as the primary endpoints. Furthermore, the independent prognostic factors of patients with the surgical intervention were retrospectively analyzed.

Results: Patients underwent surgical intervention had better OS and LCSS than those who did not ( $\mathrm{P}=0.001$ for $\mathrm{OS} ; \mathrm{P}<0.001$ for LCSS). Meanwhile, patients who underwent surgery combined with lymph node dissection had better survival outcomes ( $\mathrm{P}<0.001$ for OS and LCSS) in the K-M analysis. For different metastatic sites, PTR was beneficial to the survival of patients with isolated lung metastases (LUM) and multiple organ metastases (MOM) (LUM: $\mathrm{P}=0.041$; MOM: $\mathrm{P}=0.003)$. As for metastatic surgery, no patients were found to benefit from resection of metastatic tumor [bone metastasis $(\mathrm{BOM})$ : $\mathrm{P}=0.696$; brain metastasis (BRM): $\mathrm{P}=0.951$; LUM: $\mathrm{P}=0.402 ; \mathrm{MOM}: \mathrm{P}=0.365$ ].
\end{abstract}

Conclusions: Surgical intervention strategies can prolong survival to some extent, depending on different sites of metastasis and highly selected patients.

Keywords: Advanced lung adenocarcinoma (LUAD); metastasis; surgical intervention; prognosis

Submitted Jun 17, 2021. Accepted for publication Aug 19, 2021.

doi: $10.21037 /$ jtd-21-997

View this article at: https://dx.doi.org/10.21037/jtd-21-997

\section{Introduction}

As one of the most prevalent malignant tumors globally, lung cancer is the primary cause of cancer-related deaths, which seriously threatens human's health and life (1). Lung adenocarcinoma (LUAD) is the most common pathological subtype of lung cancer with only $5 \%$ relative survival rate of 5 -year. About $57 \%$ patients have advanced stage and metastatic diseases (2). For early-stage lung cancer, the first treatment option is surgical resection. However, surgical intervention is generally not considered for stage IV patients. The recommended treatment strategy is systemic therapy (i.e., chemotherapy, radiation therapy, molecular

\footnotetext{
$\wedge$ ORCID: 0000-0002-6975-3583.
} 
targeted therapy, or immunotherapy) (3).

At present, it is still controversial whether to perform primary tumor resection (PTR) and/or metastatic tumor resection (MTR) for LUAD with distant metastasis. Previous studies (4-6) have shown that PTR treatment strategies combined with systemic therapy such as radiotherapy or chemotherapy are beneficial for stage IV non-small cell lung cancer (NSCLC) patients with single or several synchronous metastases. For distant metastasis, several clinical studies $(5,7,8)$ have reported that MTR may show survival benefits for patients with limited metastatic sites. But few relevant studies are found with small sample size. Therefore, it is not yet confident that MTR can improve the survival rate of patients.

The general metastatic sites of LUAD are lung, bone, brain, adrenal gland, pleura, liver (9). Fewer studies are found concerning whether more significant survival benefits are provided by the surgical interventions (PTR and/or MTR) to patients with metastatic LUAD. To address this unresolved problem, we re-evaluated the significance of surgical intervention in stage IV LUAD patients registered within the Surveillance, Epidemiology, and End Results (SEER) database.

We present the following article in accordance with the STROBE reporting checklist (available at https://dx.doi. org/10.21037/jtd-21-997).

\section{Methods}

\section{Patients and data collection}

The study was conducted in accordance with the Declaration of Helsinki (as revised in 2013). The population of this retrospective study was selected from the SEER database of the National Cancer Institute, a cancer registry program that collected tumor-related data and covered approximately $28 \%$ of the U.S. population (10). The SEER data were publicly available for studies of cancer-based epidemiology and survival analyses. We had been granted access to the data used in this study (SEER-Stat username: 25824-Nov2019).

A total of 148,587 patients diagnosed with LUAD from 2010 to 2015 were extracted through the SEER database (SEER-Stat 8.3.9). LUAD was defined on the basis of the third edition of the International Classification of Diseases for Oncology (ICD-O-3). The primary site was Lung and Bronchus, and the histological type code: 8140-8147, 8255, $8260,8310,8323,8480,8481,8490,8550$, and 8572 (11).
We reclassified the TNM stage according to the eighth edition of the American Joint Committee on Cancer (AJCC) TMN stage based on the information of "CS Tumor size" and "CS Extension". Exclusion criteria include: $<18$ years of age, unknown TNM stage, non-stage IV, surgical treatments for distant metastases other than MTR, diagnosed with more than one primary tumor, received radiotherapy.

In the end, 32,497 cases were included in this study. According to the surgical strategies, we divided eligible patients into four groups: primary tumor resection only (PTR), metastatic tumor resection only (MTR), both primary and metastatic tumor resection (PMTR). Metastatic sites were divided into (I) lung metastases only (LUM) without bone, brain, or liver metastases; (II) bone metastases only (BOM) without lung, brain, or liver metastases; (III) brain metastases only (BRM) without lung, bone, or liver metastases; (IV) liver metastases only (LIM) without lung, bone, or brain metastases; (V) multiple organ metastases (MOM): two or more metastatic organs among lung, liver, brain, and bone; (VI) other metastases (OTM): no metastases to lung, bone, brain, and liver.

\section{Statistical analysis}

The chi-square test for categorical variables compared patient characteristics. Overall survival (OS) and lung cancer-specific survival (LCSS) were the primary endpoints. OS was defined as the time from the date of diagnosis to death from any cause. LCSS was the time from the date of diagnosis to specific death due to lung cancer.

A 1:1 propensity score matching (PSM) was used to reduce unbalanced baseline characteristics between the surgery and no-surgery groups. The match was conducted using the nearest-neighbor algorithm with a caliper width of 0.02 . Propensity scores were calculated using logistic regression with the following covariates: age, race, sex, location, $\mathrm{T}$ stage, $\mathrm{N}$ stage, lymph node dissection, chemotherapy, metastatic site. Similarly, we also performed 1:4 PSM for patients with surgical intervention (PTR or MTR) in different metastatic sites. $\mathrm{P}>0.05$ was regarded as an acceptable balance.

OS and LCSS were estimated by the Kaplan-Meier method and compared with the log-rank test. The multivariate Cox proportional hazards regression analysis was used to determine the independent prognostic factors of surgical intervention patients. The hazard ratio (HR) was calculated with a $95 \%$ confidence interval (CI). Statistical analysis was performed with SPSS version 23.0 (IBM, 
Armonk, NY, USA). R (version 4.1.0) was utilized to carry out the PSM. $\mathrm{P}<0.05$ was considered statistically significant.

\section{Results}

\section{Characteristics of patients}

Table 1 shows the demographic and clinicopathological characteristics of the enrolled patients. In total, 32,497 LUAD patients with distant metastases were identified. The average age at diagnosis was $67.1 \pm 11.4$ years old. The most common single metastatic site was bone (17.86\%), followed by the lung (14.16\%), brain (10.89\%), and liver (3.58\%). Nevertheless, MOM had a higher proportion, accounting for $30.86 \%$. A total of 1,363 LUAD patients underwent surgical intervention, of which $749(55.0 \%)$ patients had PTR, 548 (40.2\%) patients had MTR, and 66 (4.8\%) patients had PMTR.

\section{Impact of surgery intervention on survival outcomes in stage IV LUAD patients}

Of all eligible patients, 1,363 underwent surgical intervention, while 31,134 did not. Statistical differences in age, race, sex, location, $\mathrm{T}$ stage, $\mathrm{N}$ stage, lymph node dissection (LND), chemotherapy, and metastatic site were noted between the surgery and no-surgery groups. After PSM, all baseline

Table 1 Baseline characteristics of patients with advanced LUAD

\begin{tabular}{|c|c|c|c|c|c|c|c|}
\hline Variables & $\begin{array}{c}\text { LUM } \\
\mathrm{n}=4,600\end{array}$ & $\begin{array}{c}\text { BOM } \\
n=5,804\end{array}$ & $\begin{array}{c}\text { BRM } \\
n=3,538\end{array}$ & $\begin{array}{c}\text { LIM } \\
\mathrm{n}=1,162\end{array}$ & $\begin{array}{c}\text { MOM } \\
n=10,030\end{array}$ & $\begin{array}{c}\text { OTM } \\
\mathrm{n}=7,363\end{array}$ & $P$ value \\
\hline Age (years) & & & & & & & $<0.001$ \\
\hline $56-75$ & $2,547(55.4)$ & $3,472(59.8)$ & $2,162(61.1)$ & $694(59.7)$ & $6,179(61.6)$ & $4,098(55.7)$ & \\
\hline$\geq 76$ & $1,444(31.4)$ & $1,424(24.5)$ & $621(17.6)$ & 329 (28.3) & $2,024(20.2)$ & $2,220(30.2)$ & \\
\hline Male & $2,136(46.4)$ & $3,221(55.5)$ & $1,759(49.7)$ & $591(50.9)$ & $5,172(51.6)$ & $3,756(51.0)$ & \\
\hline Female & $2,464(53.6)$ & $2,583(44.5)$ & $1,779(50.3)$ & $571(49.1)$ & $4,858(48.4)$ & $3,607(49.0)$ & \\
\hline Race & & & & & & & $<0.001$ \\
\hline Location & & & & & & & $<0.001$ \\
\hline Main bronchus & $157(3.4)$ & $195(3.4)$ & $109(3.1)$ & $49(4.2)$ & 407 (4.1) & $305(4.1)$ & \\
\hline Upper lobe & $2,304(50.1)$ & $3,268(56.3)$ & $2,106(59.5)$ & 635 (54.6) & $5,359(53.4)$ & $3,791(51.5)$ & \\
\hline Middle lobe & $208(4.5)$ & $266(4.6)$ & $163(4.6)$ & $46(4.0)$ & $413(4.1)$ & $341(4.6)$ & \\
\hline Lower lobe & $1,171(25.5)$ & $1,571(27.1)$ & 905 (25.6) & $333(28.7)$ & $2,669(26.6)$ & $1,891(25.7)$ & \\
\hline Overlapping lesions & $71(1.5)$ & $55(0.9)$ & $19(0.5)$ & $12(1.0)$ & $92(0.9)$ & $104(1.4)$ & \\
\hline NOS & $689(15.0)$ & $449(7.7)$ & $236(6.7)$ & $87(7.5)$ & $1,090(10.9)$ & 931 (12.6) & \\
\hline
\end{tabular}

Table 1 (continued) 
Table 1 (continued)

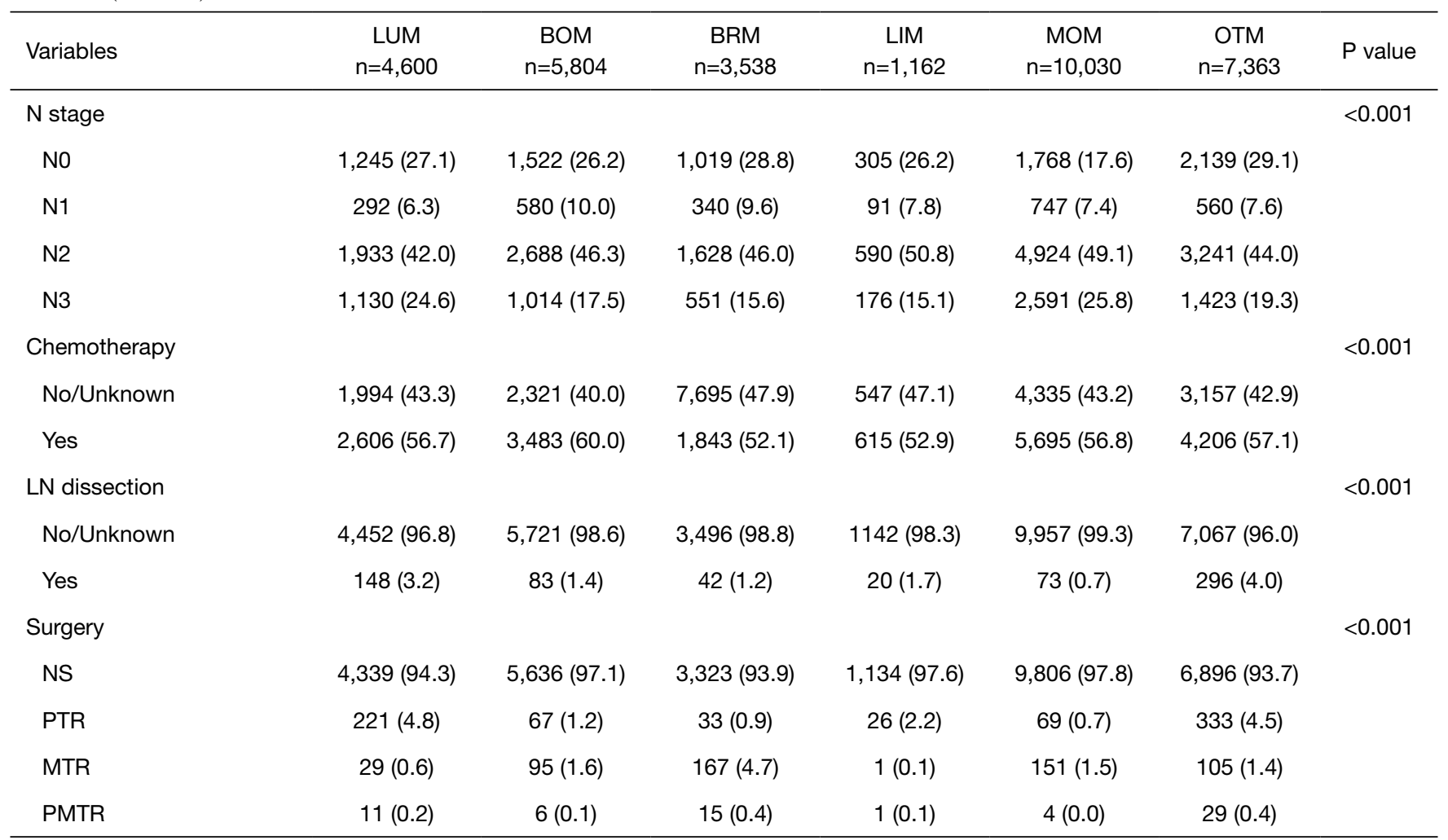

Data presented as $\mathrm{n}$ (\%). LUAD, lung adenocarcinoma; NS, no surgery; PTR, primary tumor resection only; MTR, metastatic tumor resection only; PMTR, both primary and metastatic tumor resection; LN, lymph node; LUM, lung metastases only; BOM, bone metastases only; BRM, brain metastases only; LIM, liver metastases only; MOM, multiple organ metastases; OTM, other metastases.

Table 2 Baseline characteristics of the no-surgery and surgery group before and after PSM

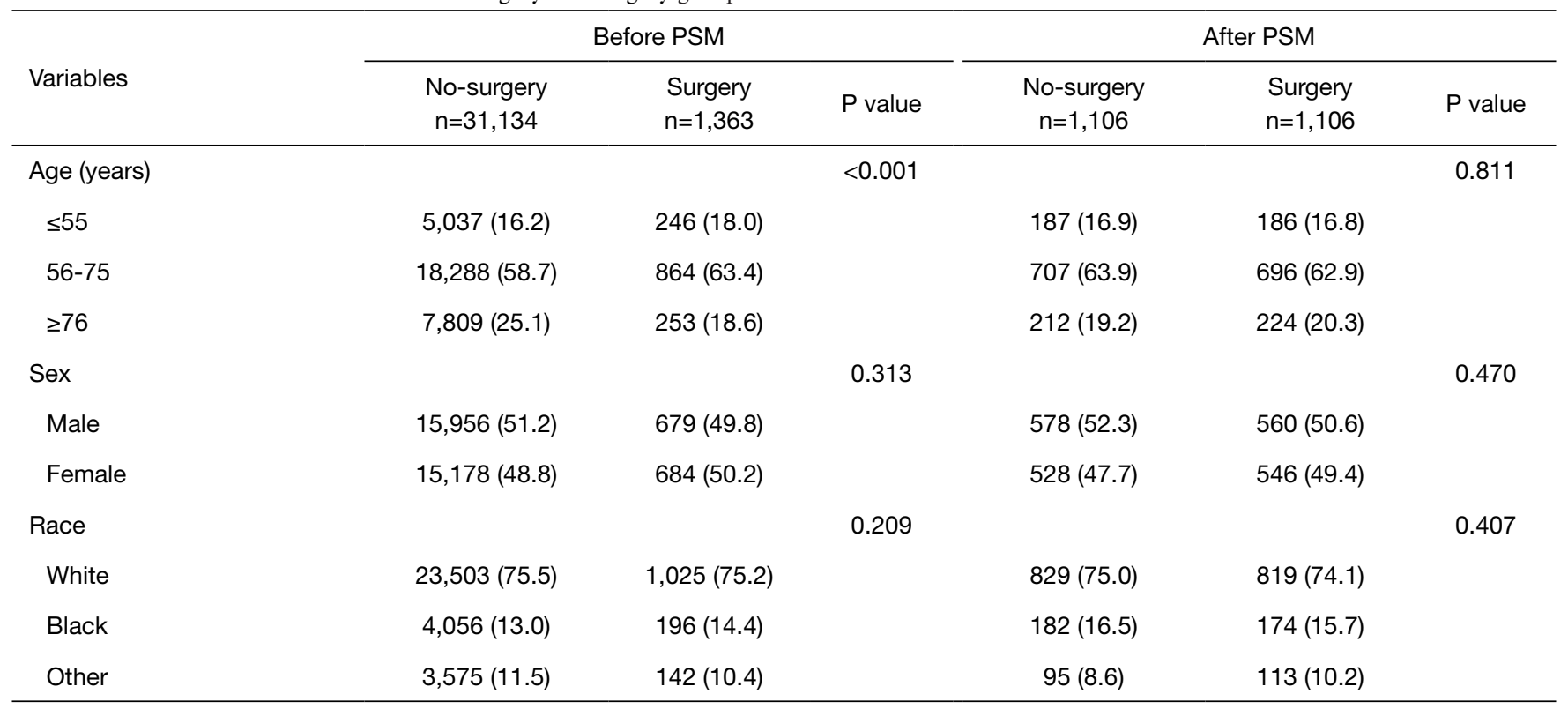

Table 2 (continued) 
Table 2 (continued)

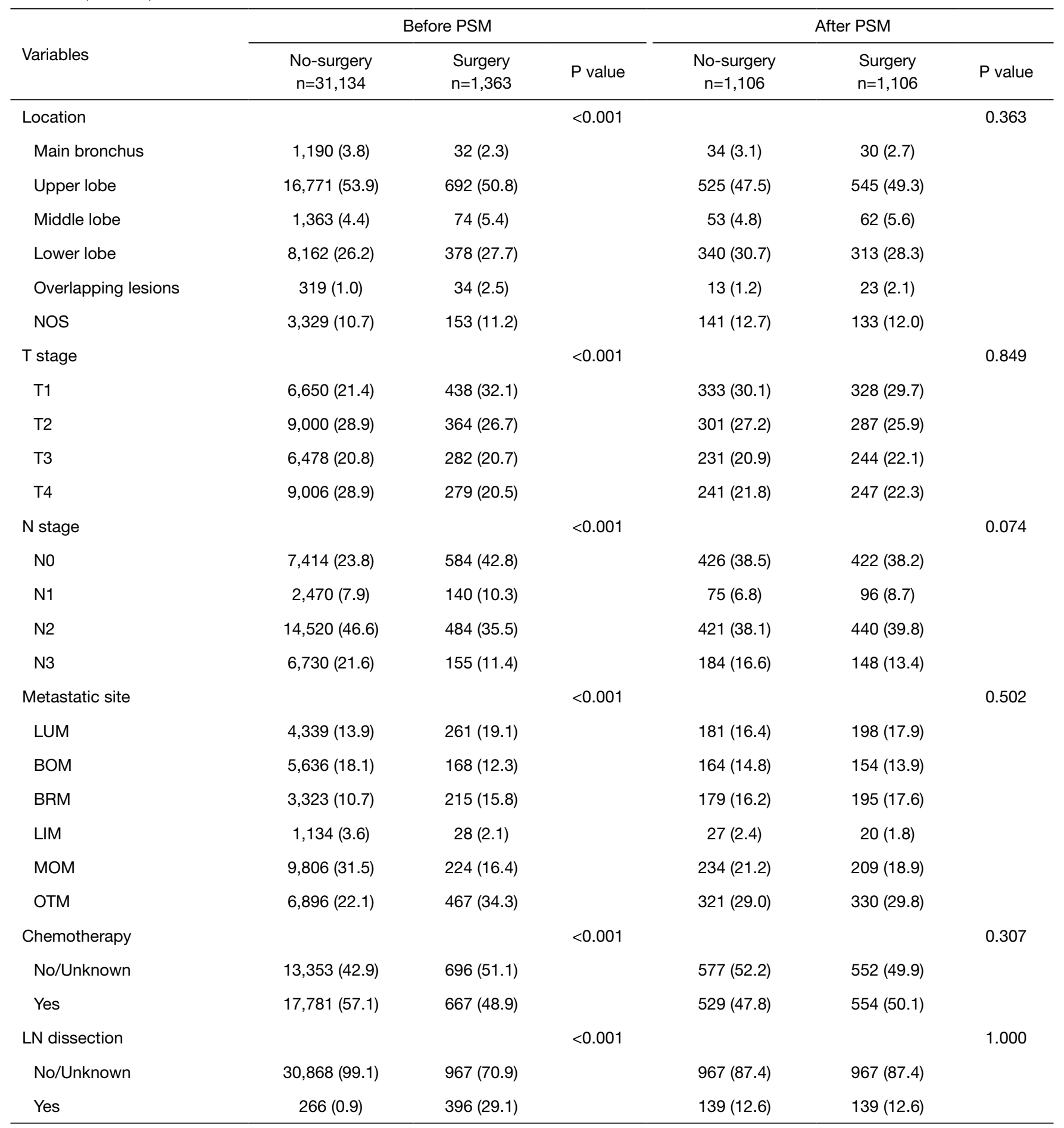

PSM, propensity score matching; LUM, lung metastases only; BOM, bone metastases only; BRM, brain metastases only; LIM, liver metastases only; MOM, multiple organ metastases; OTM, other metastases; LN, lymph node. 

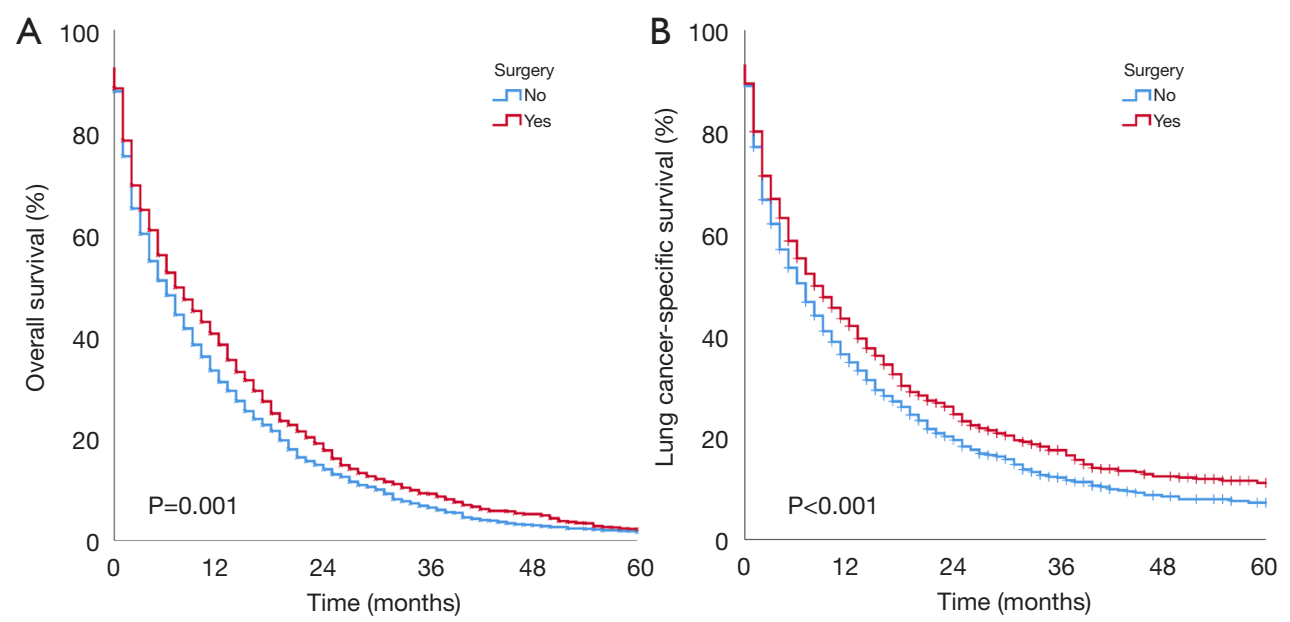

Figure 1 Kaplan-Meier curve of the no-surgery and surgery group. (A) Overall survival; (B) lung cancer-specific survival.

characteristics were well balanced ( $\mathrm{P}>0.05$; Table 2$)$.

$\mathrm{K}-\mathrm{M}$ analysis of the matched population found that patients who underwent surgical intervention had better OS and LCSS than those who did not. The median OS of the nosurgery group was 6.0 months (95\% CI: 5.3-6.7 months), and the surgery group was 7.0 months (95\% CI: 5.9-8.1 months). There was a statistical difference between the two groups $(\mathrm{P}=0.001)$ (Figure 1A). The median LCSS in the surgery group was better than the no-surgery group (surgery: 8.0 months, 95\% CI: 6.7-9.3 months; no-surgery: 7.0 months, 95\% CI: 6.1-7.9 months; $\mathrm{P}<0.001$ ) (Figure $1 B$ ).

\section{Prognostic factors for surgical intervention}

Furtherly, we analyzed the prognostic factors of patients who underwent surgery in stage IV to determine the population who would benefit from surgical intervention (Table 3). We selected the matched cohorts that underwent at least one type of surgical strategy to conduct multivariate Cox regression analysis. Compared with PTR, MTR cannot benefit patients better (HR: 1.269, 95\% CI: 1.091-1.475, $\mathrm{P}=0.002)$, and PMTR was not a prognostic factor related to OS (HR: 0.867, 95\% CI: 0.613-1.224, $\mathrm{P}=0.416$ ). Similar results also appeared in the patient's LCSS analysis. In terms of metastatic sites, LUM as a reference, BOM (HR: 1.516, 95\% CI: 1.178-1.952, P=0.001), BRM (HR: 1.356, 95\% CI: 1.048-1.754, P=0.021), LIM (HR: $1.725,95 \%$ CI: 1.022-2.911, $\mathrm{P}=0.041)$ and MOM (HR: $1.426,95 \% \mathrm{CI}$ : $1.116-1.821, \mathrm{P}=0.005)$ were adverse prognostic factors for the LCSS of patients, but isolated liver metastases (HR: $1.360,95 \%$ CI: $0.853-2.167, \mathrm{P}=0.196$ ) were not statistically related to OS. Male, advanced T stage, and $\mathrm{N}$ stage affected the patients' OS and LCSS. Meanwhile, chemotherapy and LND were favorable prognostic factors for patients.

\section{Impact of lymph node dissection on survival outcomes in stage IV LUAD patients}

According to multivariate Cox analysis in the matched population, LND was independently associated with better OS (HR: $0.767,95 \%$ CI: 0.628-0.938, $\mathrm{P}=0.010$ ) and LCSS (HR: $0.649,95 \%$ CI: 0.508-0.829, $\mathrm{P}=0.001$ ) (Table 3). We confirmed this result in the $\mathrm{K}-\mathrm{M}$ analysis (Figure 2). The median OS was 6.0 months (95\% CI: 5.1-6.9 months), and the median LCSS was 7.0 months (95\% CI: 6.0-8.0 months) in the surgery without LND group. The median OS was 18.0 months (95\% CI: 14.9-21.1 months), and the median LCSS was 20.0 months (95\% CI: 16.2-23.8 months) in the surgery with LND group. Survival outcomes were better in the patients underwent surgery combined with LND ( $\mathrm{P}<0.001$ for OS and LCSS).

\section{Correlation between different surgical intervention strategies and survival of stage IV LUAD}

OS was evaluated based on whether the primary tumor or metastatic tumor surgery was performed in patients with LUM, BOM, BRM, and MOM. Before evaluation, PSM was used to eliminate possible confounders for different surgical strategies (PTR or MTR) in different metastatic sites (Tables S1-S8). Due to the limited number, the LIM and PMTR groups were excluded from these evaluations. 
Table 3 Multivariate Cox regression analysis for OS and LCSS in patients with surgery

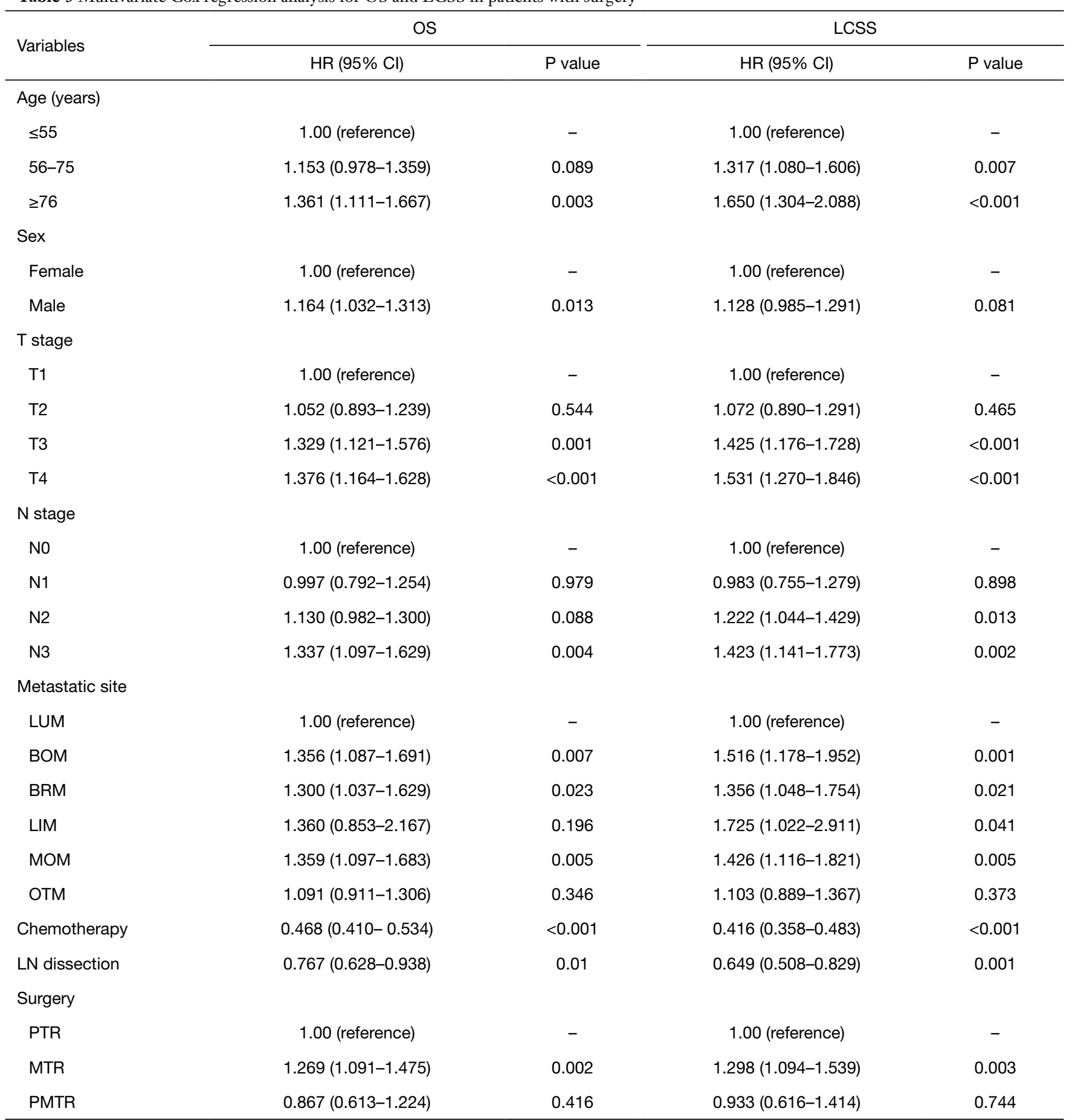

OS, overall survival; LCSS, lung cancer-specific survival; HR, hazard ratio; Cl, confidence interval; PTR, primary tumor resection only; MTR, metastatic tumor resection only; PMTR, both primary and metastatic tumor resection; LN, lymph node; LUM, lung metastases only; BOM, bone metastases only; BRM, brain metastases only; LIM, liver metastases only; MOM, multiple organ metastases; OTM, other metastases. 

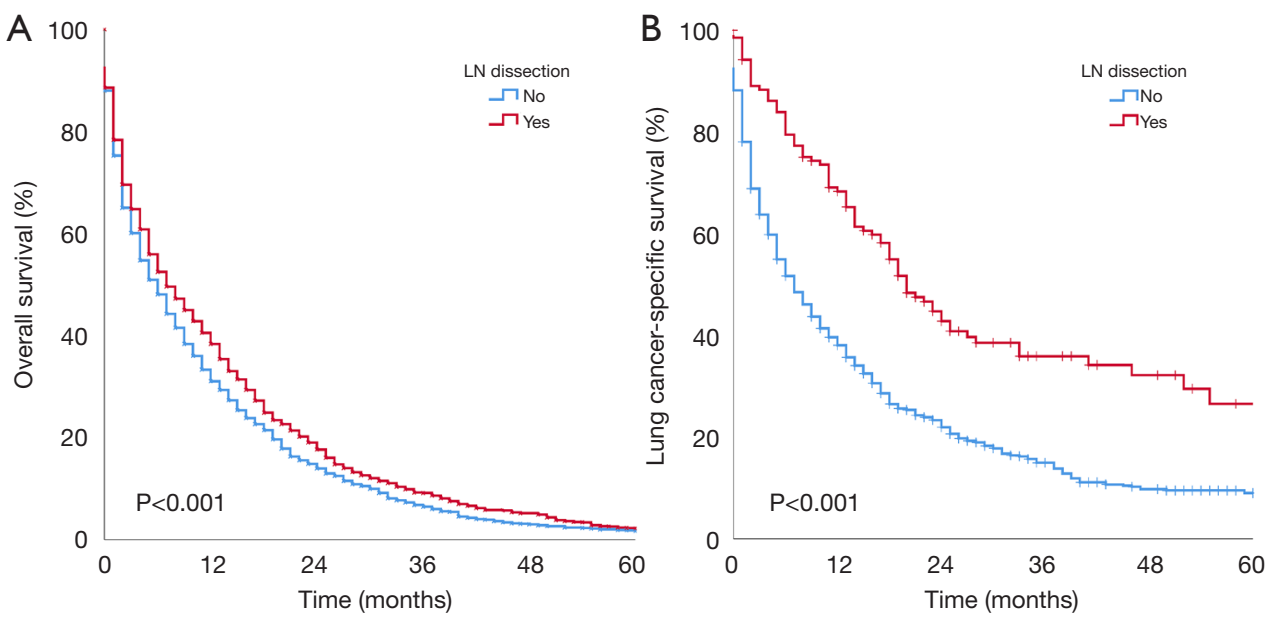

Figure 2 Kaplan-Meier curve based on whether or not lymph node dissection was performed. (A) Overall survival; (B) lung cancer-specific survival. LN, lymph node.

Patients with LUM and MOM benefited from PTR (LUM: $\mathrm{P}=0.041$; MOM: $\mathrm{P}=0.003$ ), while no survival benefits were observed for BOM and BRM (BOM: $\mathrm{P}=0.088$; BRM: $\mathrm{P}=0.124$ ) (Figure 3). On the other hand, no survival benefits were observed in MTR (LUM: $\mathrm{P}=0.402$; BOM: $\mathrm{P}=0.696$; BRM: $\mathrm{P}=0.951 ; \mathrm{MOM}: \mathrm{P}=0.365$ ) (Figure 4).

\section{Discussion}

With advances in preoperative management and surgical techniques, the mortality rate associated with surgery has been declining. In the context of constant updates in systemic treatment, increasing awareness of clinical decision-making through multidisciplinary teams (MDT) also led to re-consideration of surgical intervention as part of treatment to advanced LUAD (12). In recent years, some evidence $(3,13-15)$ supported the value of surgical intervention in patients with advanced NSCLC. Our study found that the surgery group had better OS and LCSS than the no-surgery group and that surgery combined with necessary LND prolonged the patient's survival significantly. These results may be related to the reduction of tumor burden in patients with advanced LUAD by surgical intervention on the basis of comprehensive treatment.

The most common single metastatic sites were bone, lung, brain, and liver in our data. In addition, MOM was more common than single-organ metastases. We reassessed the significance of PTR or MTR for different metastatic sites. Although the SEER database is not available for the specific number of metastatic lesions in a single metastatic organ, its considerable sample size helps us explain the effect of metastatic organ distribution on postoperative survival.

A study analyzing 1,206 patients who underwent PTR suggested that local tumor control strategies for NSCLC patients with distant metastases appear to prolong survival (4). Yang et al. (14) identified that receiving PTR is a prognostic factor for stage IV NSCLC. Local treatment of limited primary lung tumors is strongly associated with improved long-term survival (15). We found that surgical interventions for patients with LUM and MOM could prolong survival time to a certain extent. However, the effect was not significant for patients with BRM and BOM. Due to the small sample size and the possible confounding factors, prospective studies should be conducted in the future.

Previously, some single-center studies (16-18) demonstrated that surgical management of patients with oligometastatic NSCLC to the brain is associated with excellent long-term survival. Daniels et al. (18) retrospectively analyzed 15 NSCLC patients who underwent BRM resection, and 12 patients were resected entirely. Surprisingly, the 5-year OS after attempted radical resection of BRM and successful complete resection was $60 \%$ and $70 \%$, similar to those only need to resect lung cancer completely. Nevertheless, in another study (19), 86 patients with metachronous BRM underwent surgery or radiation of their oligometastatic intracranial disease had a less 5 -year survival rate of $22 \%$, which indicated 

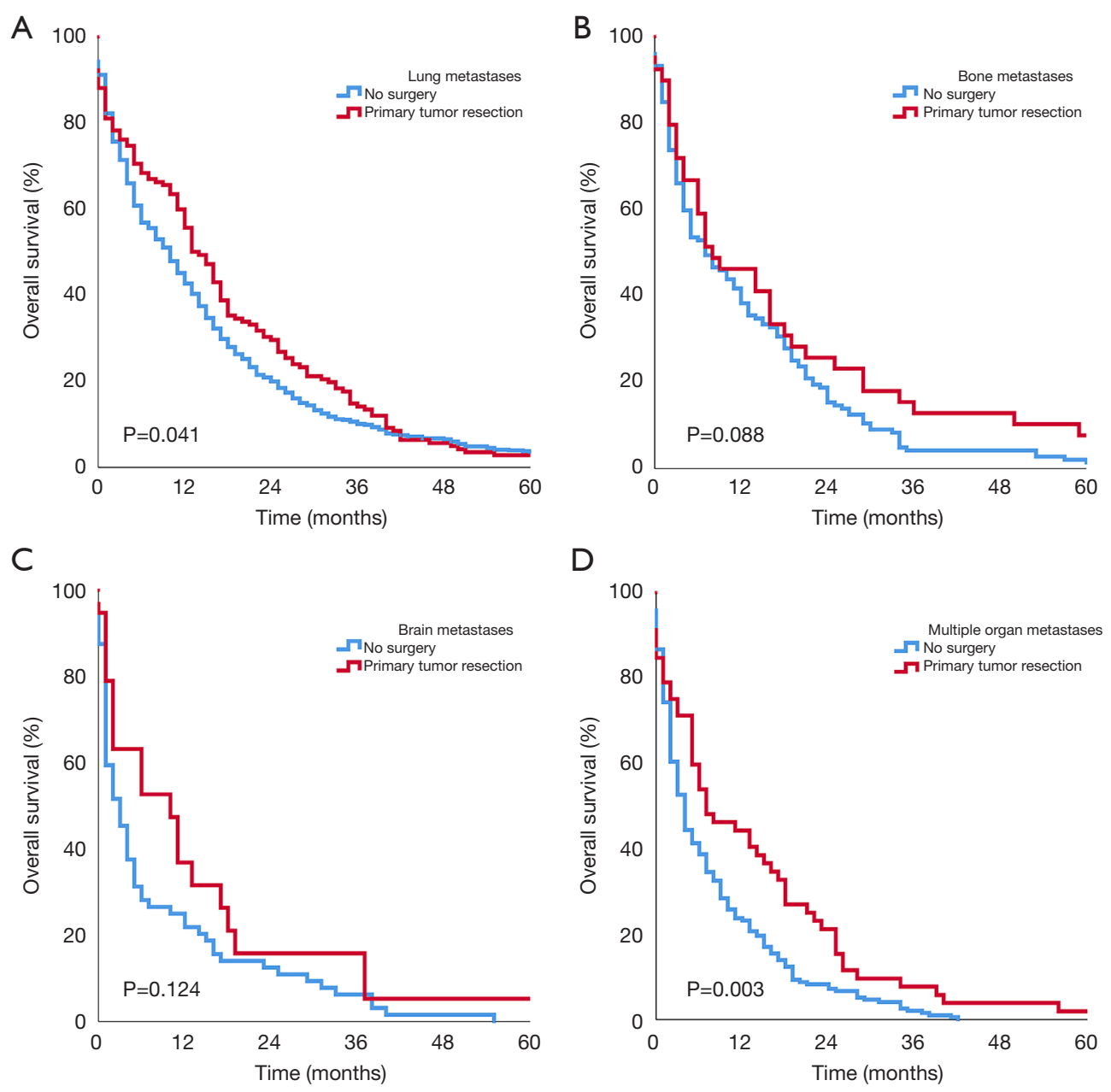

Figure 3 Kaplan-Meier curve of overall survival based on whether or not primary tumor resection was performed. (A) Patients with isolated lung metastases; (B) patients with isolated bone metastases; (C) patients with isolated brain metastases; (D) patients with multiple organ metastases.

that surgical intervention for patients with intracranial metastases might result in diminished survival. This study found that patients with BRM did not benefit from surgical resection. In clinical practice, most BRM of lung cancer choose gamma knife treatment locally. Given that radiotherapy cannot be well distinguished from surgery, we excluded patients treated with radiotherapy. The effects of radiotherapy on BRM are better than surgical resection with fewer postoperative complications (20).

There is no consensus on treating isolated metastatic diseases involving bone metastases, contralateral lung metastases, or other organ metastases $(6,17,21-24)$. Patients with LUM, BOM, or MOM did not benefit from MTR in our study. We believe that surgical intervention for metastases without resection of the primary tumor cannot fundamentally reduce the tumor burden of patients, nor can the potential risk of metastasis and recurrence be excluded. Therefore, MTR is not recommended for general use. Unfortunately, the number of patients who underwent PMTR was relatively small, so we could not analyze these patients further. But in the multivariate Cox proportional hazards regression analysis, PMTR was not an independent risk factor for patients in the surgery cohort, which is related to the impact of multi-organ surgery on patients' poor physical state and postoperative complications.

Currently, the NCCN guidelines suggest that patients with single brain or adrenal metastasis, but the primary tumor lesion is limited, local treatment for metastatic NSCLC lesions followed by resection of the primary tumor is recommended (3). A growing number of practice 

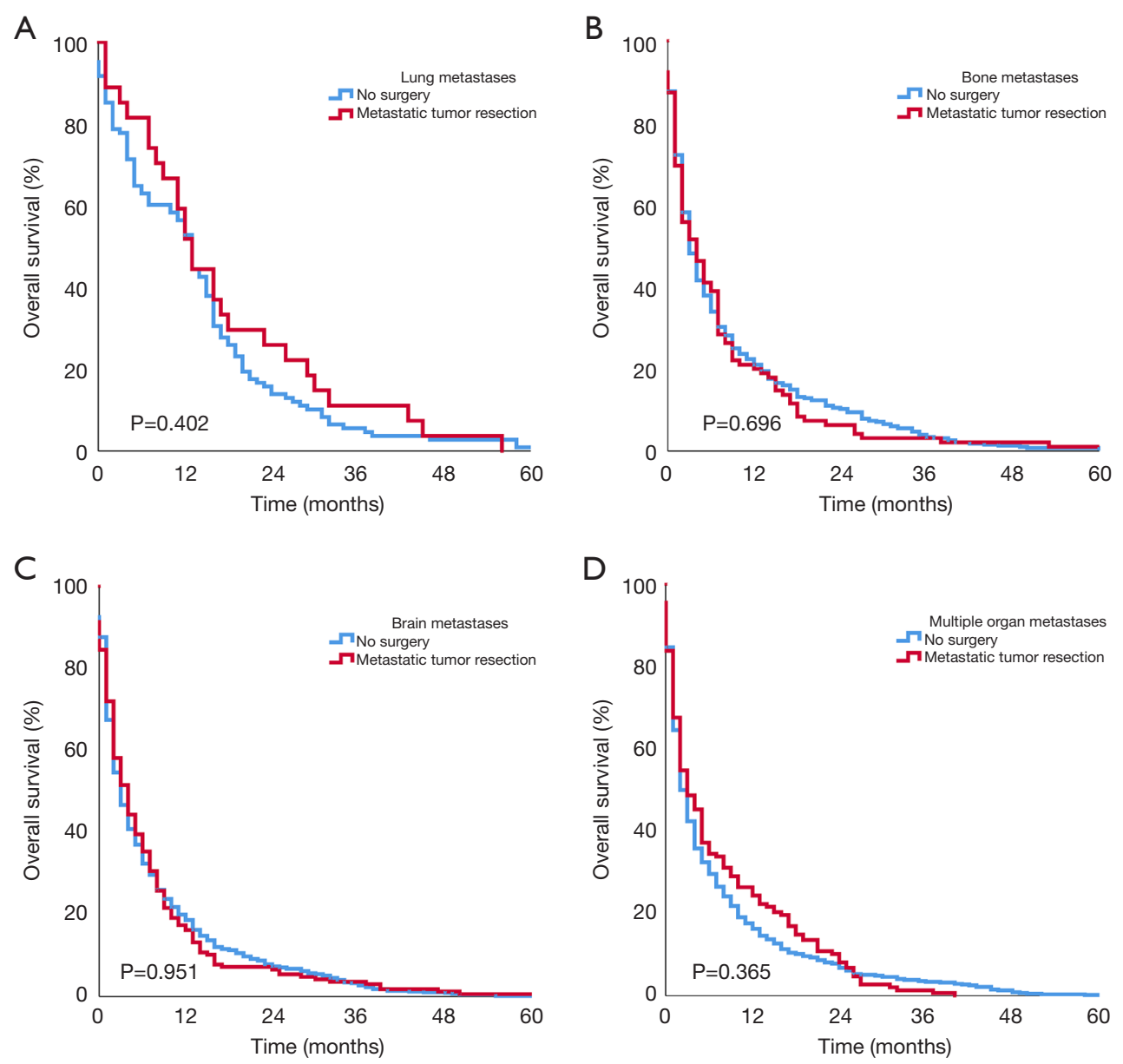

Figure 4 Kaplan-Meier curve of overall survival based on whether or not metastatic tumor resection was performed. (A) Patients with isolated lung metastases; (B) patients with isolated bone metastases; (C) patients with isolated brain metastases; (D) patients with multiple organ metastases.

guidelines have revised the recommendation for PTR as a treatment option for stage IV disease, especially those with limited extrathoracic metastatic disease and early-stage lung tumors. The reality is that few patients receive surgical interventions, and surgery in clinical practice often depends on the surgeon's judgment or the subjective consent of patients (4). Lack of standardized and systematic selection of individualized treatment is another reason that patients with LUM, BOM, BRM, or MOM could not benefit from MTR surgery.

As far as we know, there has been limited study with the primary aim to the comparison of the survival outcomes of LUAD patients with surgical intervention strategies for different metastatic sites. Based on our analysis, we believe that surgical intervention should not be rejected outright for advanced LUAD but should be carried out when necessary under the careful selection of patients to improve the survival outcomes of some patients. Females, earlier $\mathrm{T}$ stage and $\mathrm{N}$ stage, chemotherapy, and LND were more likely to benefit from surgical intervention strategies.

Although this analysis suggests survival benefit of surgical intervention strategies for patients with stage IV LUAD, there are several unavoidable limitations in the study, most of which are attributed to the insufficient information in the SEER database: (I) The database cannot list how many metastatic lesions are in a single organ, so it is impossible to study the impact of the metastasis number. (II) The study excluded patients with radiotherapy who influence the assessment of the surgical intervention. Thus, the role of radiotherapy may be overlooked. (III) Because the information of metastatic organs other than lung, bone, brain, and liver is missing, the impact of 
surgery on other organs cannot be accurately assessed. (IV) Another limitation of the current research is that data on systemic therapies are unavailable; target therapies and immunotherapy, for example. Nonetheless, as the only comprehensive population-based database, the SEER database provides the ideal approach to study the survival of these patients with open access worldwide. Prospective randomized controlled trials with large sample sizes are needed to further validate the survival benefits of surgical intervention in patients with metastatic LUAD.

\section{Conclusions}

The poor prognosis of metastatic LUAD has prompted thoracic surgeons and oncologists to seek and evaluate a variety of treatment strategies to extend survival. This study demonstrates that surgical intervention may be an option to improve the survival of some LUAD patients with different metastatic sites after highly individualized selection.

\section{Acknowledgments}

Funding: This study was supported by the National Natural Science Foundation of China [grant number 81972172], the Clinical Research Plan of Shanghai Hospital Development Center [grant number SHDC2020CR2020B, SHDC12018122], Shanghai Science and Technology Committee [grant number 19XD1423200], and Programs of Shanghai Pulmonary Hospital [grant number FKCX1904, FKLY20004].

\section{Footnote}

Reporting Checklist: The authors have completed the STROBE reporting checklist. Available at https://dx.doi. org/10.21037/jtd-21-997

Peer Review File: Available at https://dx.doi.org/10.21037/ jtd-21-997

Conflicts of Interest: All authors have completed the ICMJE uniform disclosure form (available at https:// dx.doi.org/10.21037/jtd-21-997). All authors report funding from the National Natural Science Foundation of China [grant number 81972172], the Clinical Research Plan of Shanghai Hospital Development Center [grant number SHDC2020CR2020B, SHDC12018122], the Emerging Frontier Technology Project of Shanghai
Shenkang Hospital Development Center (grant number SHDC12019130), Shanghai Science and Technology Committee [grant number 19XD1423200], and Programs of Shanghai Pulmonary Hospital [grant number FKCX1904, FKLY20004].

Ethical Statement: The authors are accountable for all aspects of the work in ensuring that questions related to the accuracy or integrity of any part of the work are appropriately investigated and resolved. The study was conducted in accordance with the Declaration of Helsinki (as revised in 2013). The requirement for informed consent was waived since the SEER database is publicly available and anonymous. The Ethics Committee of Shanghai Pulmonary Hospital, Tongii University School of Medicine, Shanghai, China, approved this study as exempted research with no human subject involved.

Open Access Statement: This is an Open Access article distributed in accordance with the Creative Commons Attribution-NonCommercial-NoDerivs 4.0 International License (CC BY-NC-ND 4.0), which permits the noncommercial replication and distribution of the article with the strict proviso that no changes or edits are made and the original work is properly cited (including links to both the formal publication through the relevant DOI and the license). See: https://creativecommons.org/licenses/by-nc-nd/4.0/.

\section{References}

1. Bray F, Ferlay J, Soerjomataram I, et al. Global cancer statistics 2018: GLOBOCAN estimates of incidence and mortality worldwide for 36 cancers in 185 countries. CA Cancer J Clin 2018;68:394-424.

2. Zhao C, Liu J, Zhou H, et al. NEIL3 may act as a potential prognostic biomarker for lung adenocarcinoma. Cancer Cell Int 2021;21:228.

3. Ettinger DS, Aisner DL, Wood DE, et al. NCCN Guidelines Insights: Non-Small Cell Lung Cancer, Version 5.2018. J Natl Compr Canc Netw 2018;16:807-21.

4. Sun Z, Sui X, Yang F, et al. Effects of primary tumor resection on the survival of patients with stage IV extrathoracic metastatic non-small cell lung cancer: A population-based study. Lung Cancer 2019;129:98-106.

5. Abdel-Rahman O. Outcomes of Surgery as Part of the Management of Metastatic Non-Small-Cell Lung Cancer: A Surveillance, Epidemiology and End Results Database Analysis. Cancer Invest 2018;36:238-45. 
6. David EA, Clark JM, Cooke DT, et al. The Role of Thoracic Surgery in the Therapeutic Management of Metastatic Non-Small Cell Lung Cancer. J Thorac Oncol 2017;12:1636-45.

7. Mordant P, Arame A, Foucault C, et al. Surgery for metastatic pleural extension of non-small-cell lung cancer. Eur J Cardiothorac Surg 2011;40:1444-9.

8. Bougie E, Masson-Côté L, Mathieu D. Comparison Between Surgical Resection and Stereotactic Radiosurgery in Patients with a Single Brain Metastasis from Non-Small Cell Lung Cancer. World Neurosurg 2015;83:900-6.

9. Klikovits T, Lohinai Z, Fábián K, et al. New insights into the impact of primary lung adenocarcinoma location on metastatic sites and sequence: A multicenter cohort study. Lung Cancer 2018;126:139-48.

10. Doll KM, Rademaker A, Sosa JA. Practical Guide to Surgical Data Sets: Surveillance, Epidemiology, and End Results (SEER) Database. JAMA Surg 2018;153:588-9.

11. Morgensztern D, Waqar S, Subramanian J, et al. Prognostic significance of tumor size in patients with stage III non-small-cell lung cancer: a surveillance, epidemiology, and end results (SEER) survey from 1998 to 2003. J Thorac Oncol 2012;7:1479-84.

12. Pillay B, Wootten AC, Crowe H, et al. The impact of multidisciplinary team meetings on patient assessment, management and outcomes in oncology settings: A systematic review of the literature. Cancer Treat Rev 2016;42:56-72.

13. David EA, Canter RJ, Chen Y, et al. Surgical Management of Advanced Non-Small Cell Lung Cancer Is Decreasing But Is Associated With Improved Survival. Ann Thorac Surg 2016;102:1101-9.

14. Yang CJ, Gu L, Shah SA, et al. Long-term outcomes of surgical resection for stage IV non-small-cell lung cancer: A national analysis. Lung Cancer 2018;115:75-83.

15. Ashworth A, Rodrigues G, Boldt G, et al. Is there an oligometastatic state in non-small cell lung cancer?

Cite this article as: Zhu S, Ge T, Hu J, Jiang G, Zhang P. Prognostic value of surgical intervention in advanced lung adenocarcinoma: a population-based study. J Thorac Dis 2021;13(10):5942-5953. doi: 10.21037/jtd-21-997
A systematic review of the literature. Lung Cancer 2013;82:197-203.

16. Gray PJ, Mak RH, Yeap BY, et al. Aggressive therapy for patients with non-small cell lung carcinoma and synchronous brain-only oligometastatic disease is associated with long-term survival. Lung Cancer 2014;85:239-44.

17. Congedo MT, Cesario A, Lococo F, et al. Surgery for oligometastatic non-small cell lung cancer: longterm results from a single center experience. J Thorac Cardiovasc Surg 2012;144:444-52.

18. Daniels M, Wright GM. Complete resection of non-smallcell lung cancer and oligo-metastatic brain disease. ANZ J Surg 2005;75:963-6.

19. Bae MK, Yu WS, Byun GE, et al. Prognostic factors for cases with no extracranial metastasis in whom brain metastasis is detected after resection of non-small cell lung cancer. Lung Cancer 2015;88:195-200.

20. Skeie BS, Eide GE, Flatebø M, et al. Quality of life is maintained using Gamma Knife radiosurgery: a prospective study of a brain metastases patient cohort. J Neurosurg 2017;126:708-25.

21. Mercier O, Fadel E, de Perrot M, et al. Surgical treatment of solitary adrenal metastasis from non-small cell lung cancer. J Thorac Cardiovasc Surg 2005;130:136-40.

22. Hanagiri T, Takenaka M, Oka S, et al. Results of a surgical resection for patients with stage IV non--small-cell lung cancer. Clin Lung Cancer 2012;13:220-4.

23. Collaud S, Stahel R, Inci I, et al. Survival of patients treated surgically for synchronous single-organ metastatic NSCLC and advanced pathologic TN stage. Lung Cancer 2012;78:234-8.

24. Salah S, Tanvetyanon T, Abbasi S. Metastatectomy for extra-cranial extra-adrenal non-small cell lung cancer solitary metastases: systematic review and analysis of reported cases. Lung Cancer 2012;75:9-14. 


\section{Supplementary}

Table S1 Baseline characteristics of patients with lung metastases underwent PTR before and after PSM

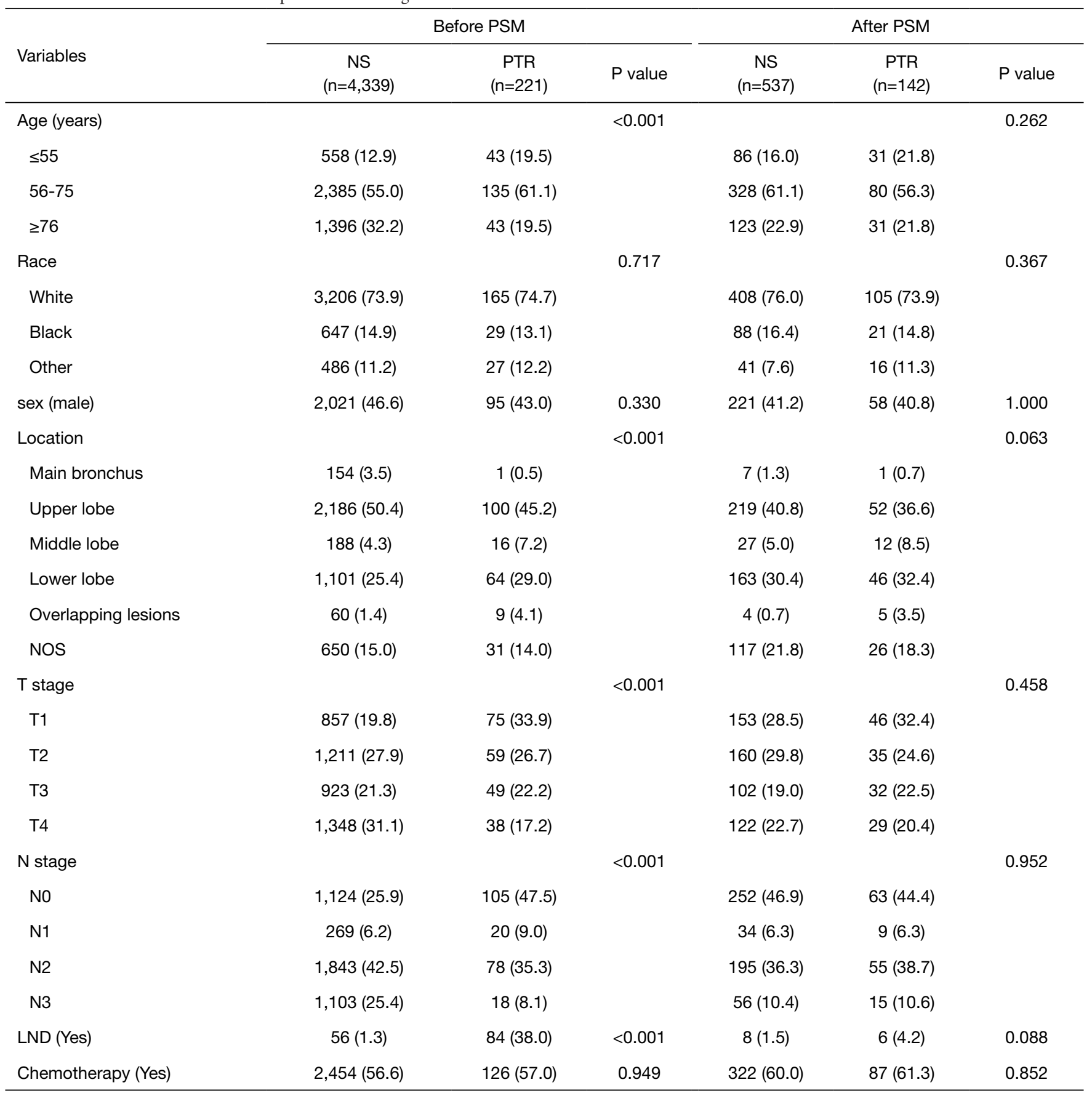

NS, no surgery; PTR, primary tumor resection only; LND, lymph node dissection. 
Table S2 Baseline characteristics of patients with bone metastases underwent PTR before and after PSM

\begin{tabular}{|c|c|c|c|c|c|c|}
\hline Variables & \multicolumn{3}{|c|}{ Before PSM } & \multicolumn{3}{|c|}{ After PSM } \\
\hline Age (years) & & & 0.053 & & & 0.824 \\
\hline$\leq 55$ & $883(15.7)$ & $13(19.4)$ & & $34(23.6)$ & $9(23.1)$ & \\
\hline Race & & & 0.496 & & & 0.874 \\
\hline White & 4,421 (78.4) & $53(79.1)$ & & $116(80.6)$ & $30(76.9)$ & \\
\hline Black & $674(12.0)$ & $10(14.9)$ & & $18(12.5)$ & $6(15.4)$ & \\
\hline Main bronchus & $188(3.3)$ & $3(4.5)$ & & $6(4.2)$ & $3(7.7)$ & \\
\hline Upper lobe & $3,175(56.3)$ & $31(46.3)$ & & $71(49.3)$ & $16(41.0)$ & \\
\hline Middle lobe & $257(4.6)$ & $6(9.0)$ & & $5(3.5)$ & $2(5.1)$ & \\
\hline Lower lobe & $1,530(27.1)$ & $21(31.3)$ & & $54(37.5)$ & $13(33.3)$ & \\
\hline Overlapping lesions & $53(0.9)$ & $2(3.0)$ & & $1(0.7)$ & $1(2.6)$ & \\
\hline NOS & $433(7.7)$ & $4(6.0)$ & & $7(4.9)$ & $4(10.3)$ & \\
\hline T stage & & & 0.362 & & & 0.990 \\
\hline No & 1,457 (25.9) & $27(40.3)$ & & $53(36.8)$ & $14(35.9)$ & \\
\hline $\mathrm{N} 1$ & $562(10.0)$ & $7(10.4)$ & & $17(11.8)$ & $3(7.7)$ & \\
\hline N2 & 2,622 (46.5) & $24(35.8)$ & & $48(33.3)$ & $14(35.9)$ & \\
\hline N3 & 995 (17.7) & $9(13.4)$ & & $26(18.1)$ & $8(20.5)$ & \\
\hline LND (Yes) & $46(0.8)$ & $32(47.8)$ & $<0.001$ & $4(2.8)$ & $4(10.3)$ & 0.113 \\
\hline Chemotherapy (Yes) & $3,397(60.3)$ & $47(70.1)$ & 0.129 & $95(66.0)$ & $26(66.7)$ & 1.000 \\
\hline
\end{tabular}

NS, no surgery; PTR, primary tumor resection only; LND, lymph node dissection. 
Table S3 Baseline characteristics of patients with brain metastases underwent PTR before and after PSM

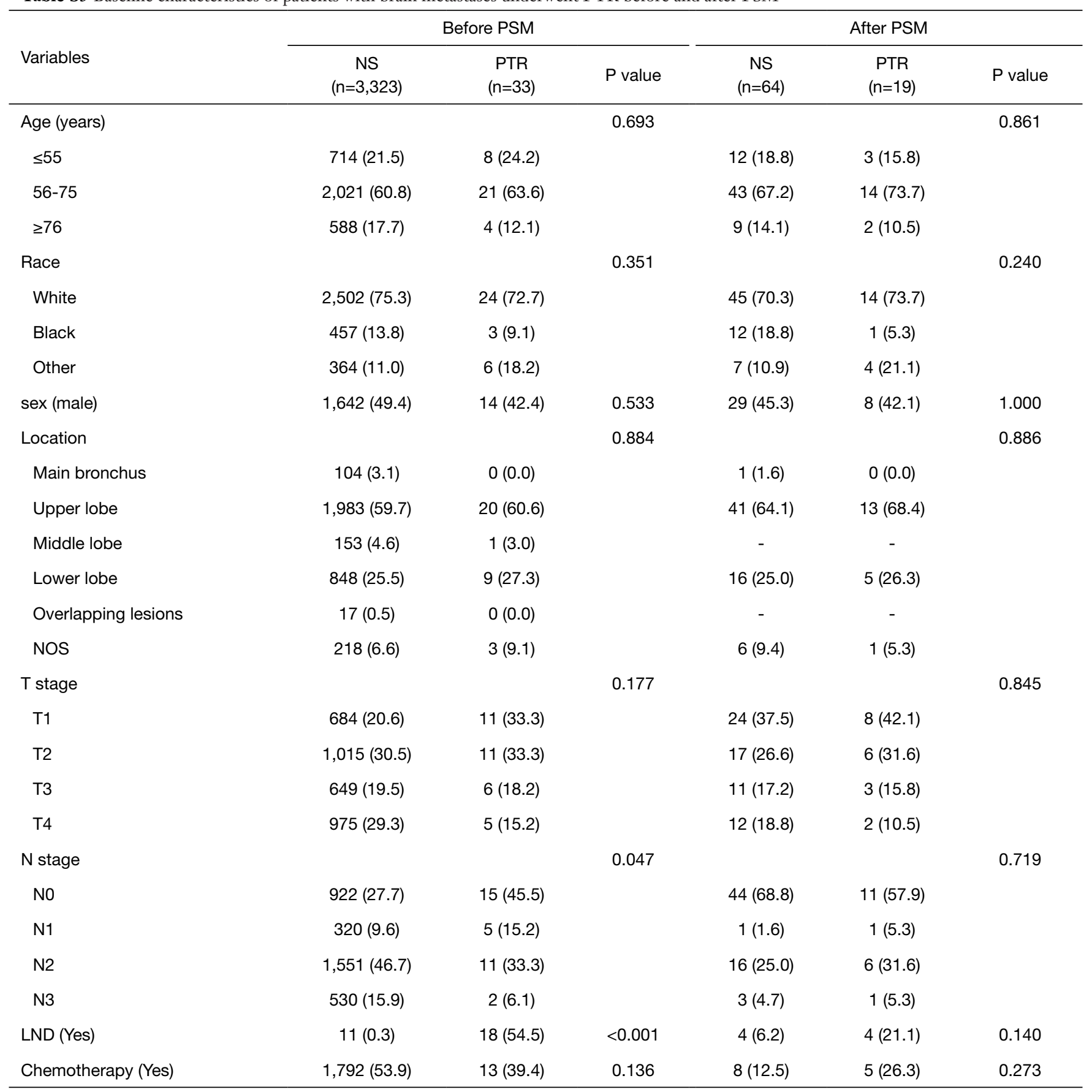

NS, no surgery; PTR, primary tumor resection only; LND, lymph node dissection. 
Table S4 Baseline characteristics of patients with multiple organ metastases underwent PTR before and after PSM

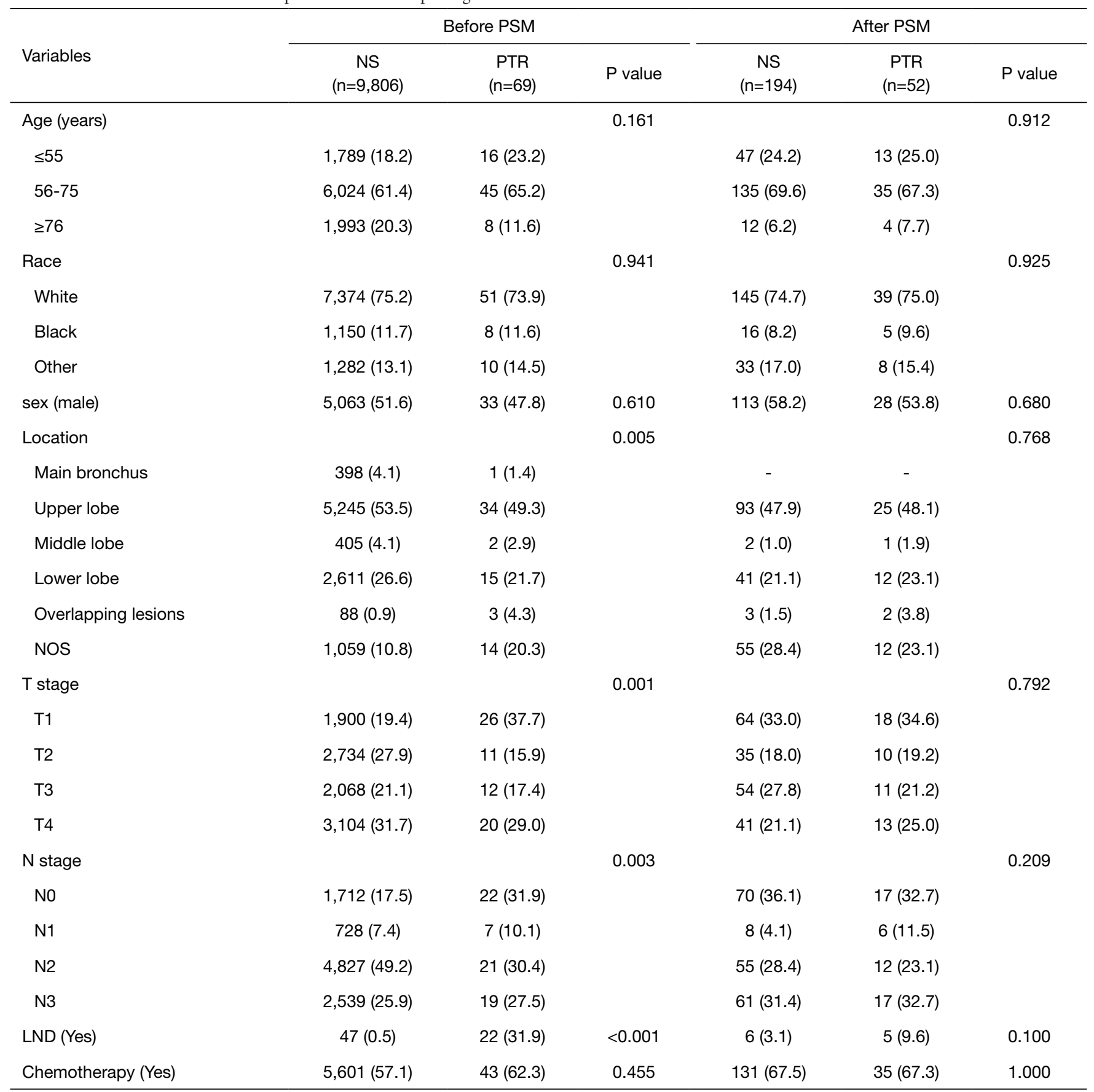

NS, no surgery; PTR, primary tumor resection only; LND, lymph node dissection. 
Table S5 Baseline characteristics of patients with lung metastases underwent MTR before and after PSM

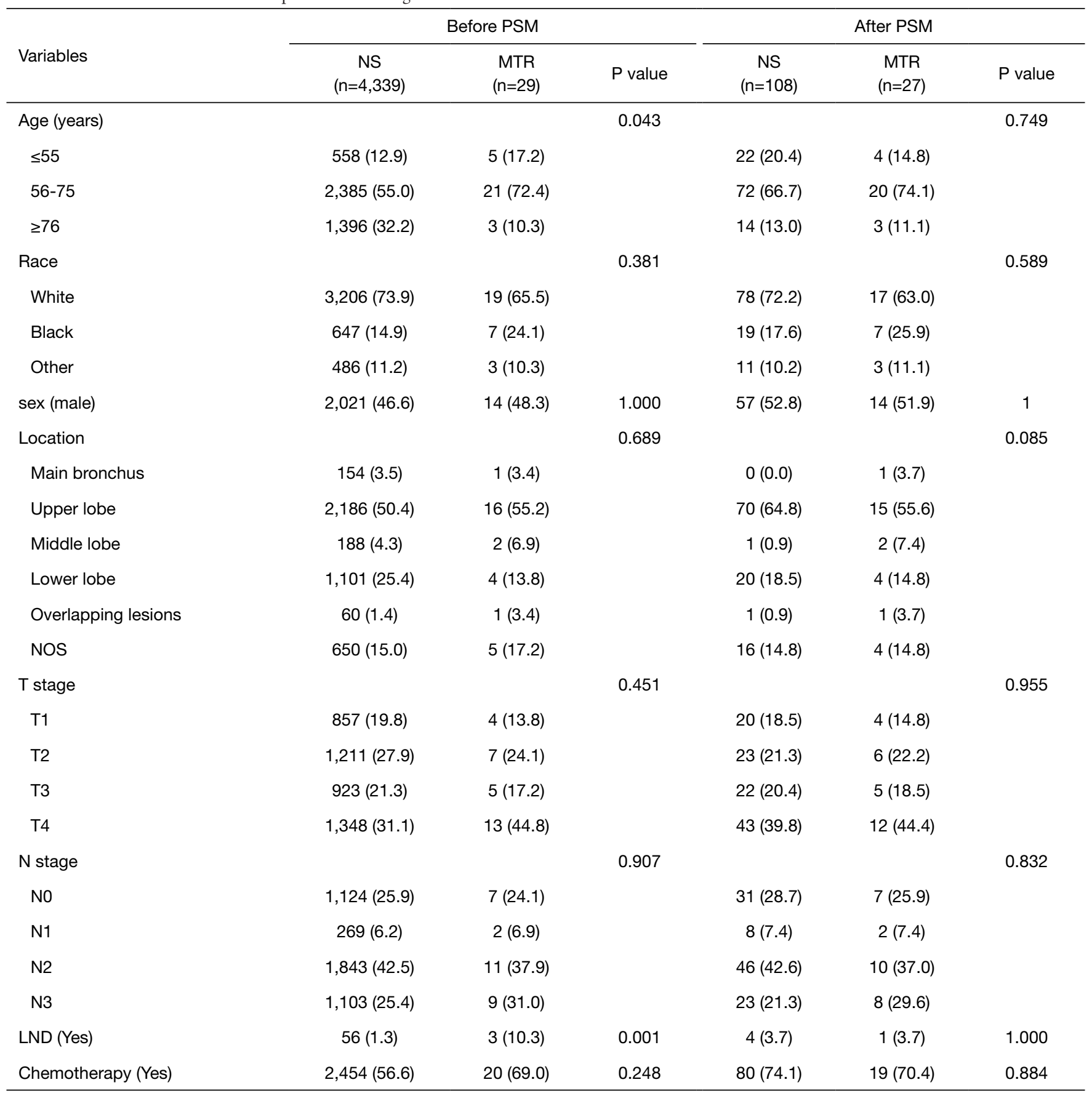

NS, no surgery; MTR, metastatic tumor resection only; LND, lymph node dissection. 
Table S6 Baseline characteristics of patients with bone metastases underwent MTR before and after PSM

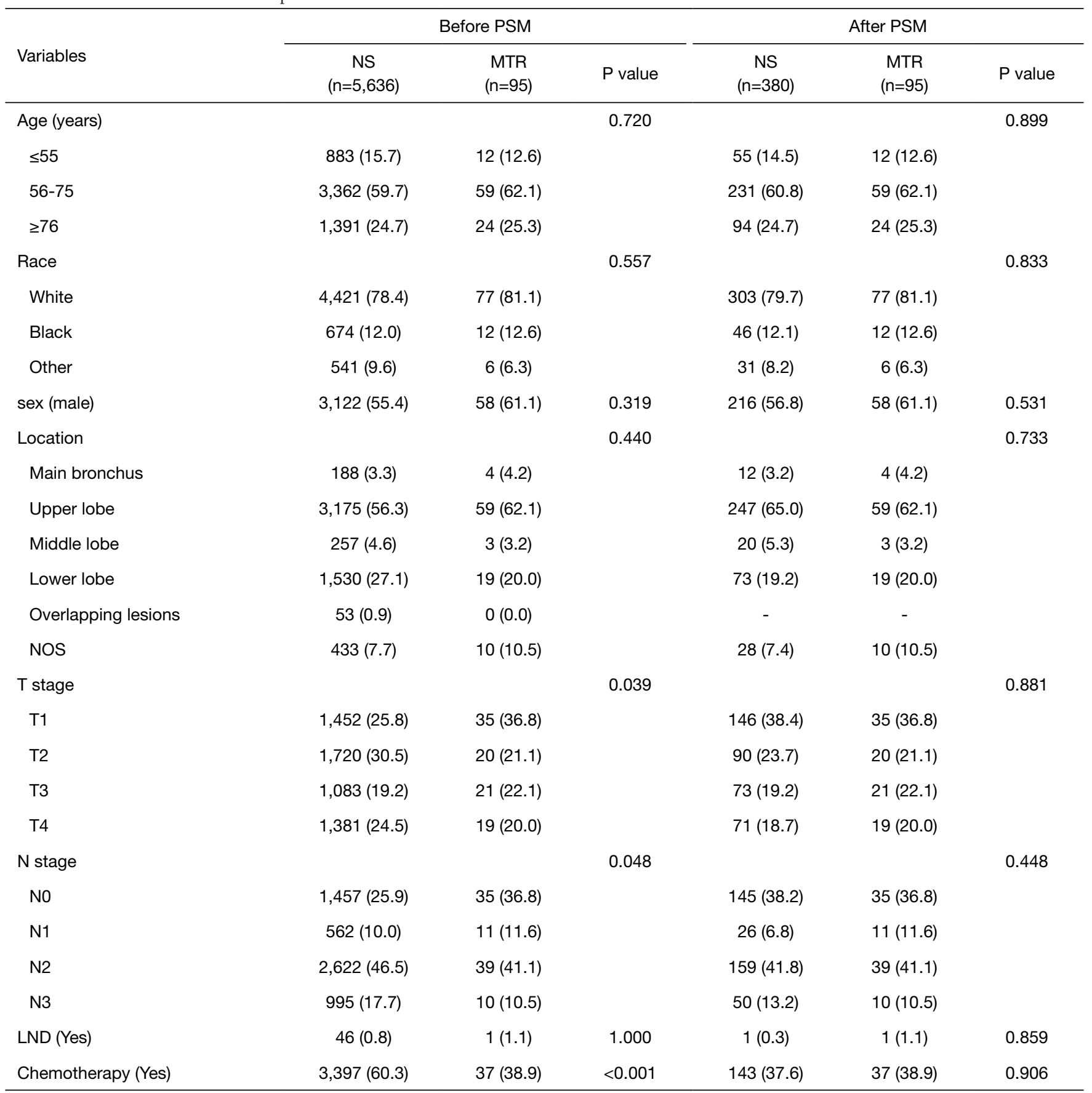

NS, no surgery; MTR, metastatic tumor resection only; LND, lymph node dissection. 
Table S7 Baseline characteristics of patients with brain metastases underwent MTR before and after PSM

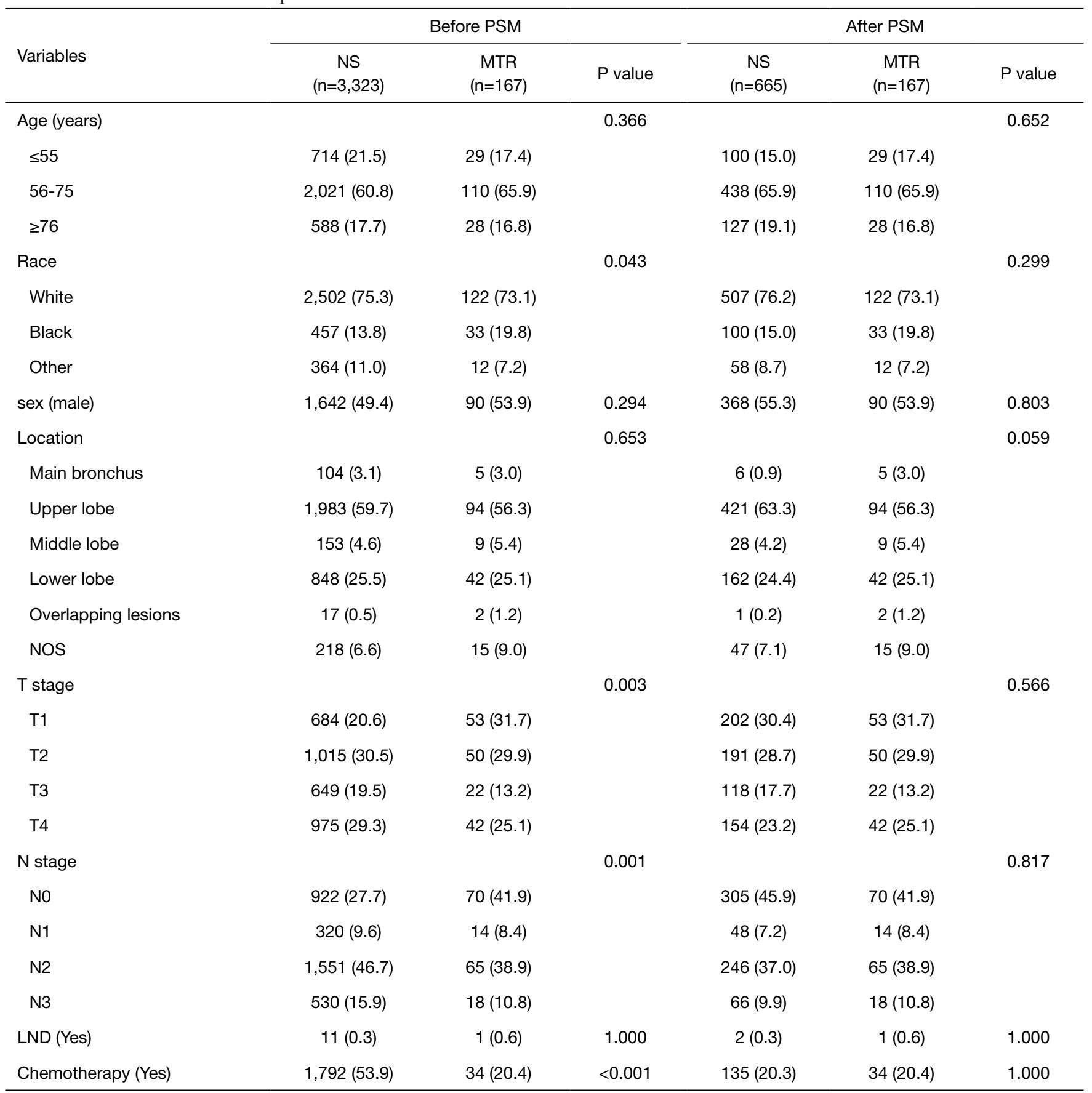

NS, no surgery; MTR, metastatic tumor resection only; LND, lymph node dissection. 
Table S8 Baseline characteristics of patients with multiple organ metastases underwent MTR before and after PSM

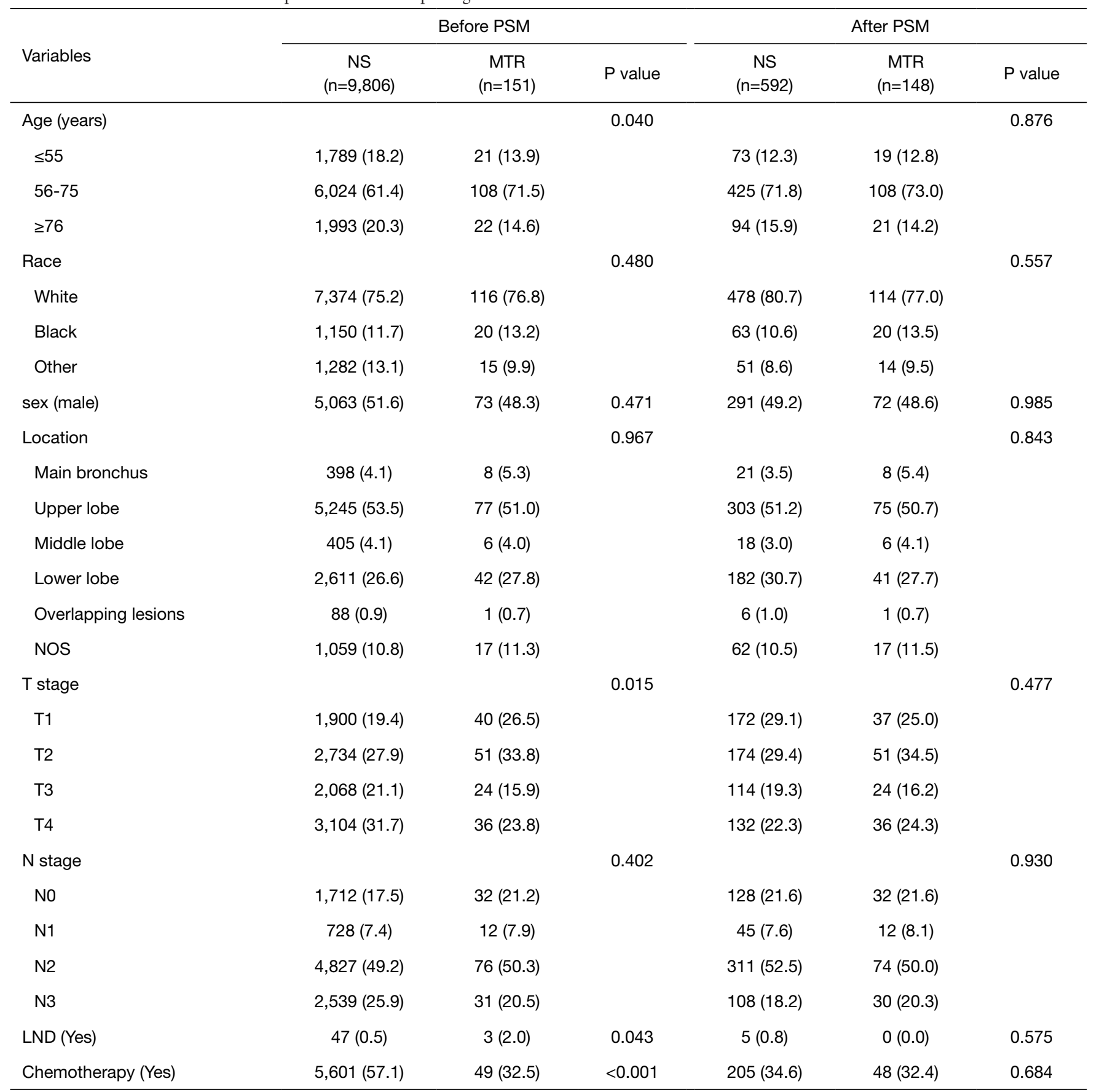

NS, no surgery; MTR, metastatic tumor resection only; LND, lymph node dissection. 